\title{
Comparison of five staging systems in hepatocellular carcinoma treated with sorafenib: A single-center experience
}

\author{
MASATSUGU ENDO*, HIROKI NISHIKAWA*, RYUICHI KITA, TORU KIMURA, YOSHIAKI OHARA, \\ AZUSA SAKAMOTO, SUMIO SAITO, NORIHIRO NISHIJIMA, AKIHIRO NASU, \\ HIDEYUKI KOMEKADO and YUKIO OSAKI
}

Department of Gastroenterology and Hepatology, Osaka Red Cross Hospital, Osaka, Osaka 543-0027, Japan

Received March 13, 2015; Accepted October 9, 2015

DOI: $10.3892 / \operatorname{mco} .2016 .755$

\begin{abstract}
To the best of our knowledge, none of the prognostic staging systems for hepatocellular carcinoma (HCC) patients who underwent sorafenib therapy is universally adopted or preferred. In the present study, we aimed to compare prognostic ability among five prognostic systems, including the Japan Integrated Staging (JIS) system, the Barcelona Clinic Liver Cancer classification system, the tumor-node-metastasis classification system, the Cancer of the Liver Italian Program scoring system and the Chinese University Prognostic Index (CUPI) scoring system for HCC patients who received sorafenib therapy. A total of 143 HCC patients treated with sorafenib were analysed. We compared prognostic ability among the five prognostic systems using the likelihood ratio (LR) $\chi^{2}$ test, linear trend $\chi^{2}$ test and concordance index (c-index). Our cohort included 114 men and 29 women. The median patient age was 71 years (range, $45-89$ years). A total of 102 patients were classified as Child-Pugh A and 41 as Child-Pugh B, whereas 31 patients $(21.7 \%)$ had portal vein invasion and $63(44.1 \%)$ extrahepatic metastases. The median survival time was 6.9 months. In the LR $\chi^{2}$ test, the CUPI scoring system had the highest value (35.804), followed by the JIS system (17.469). In the linear trend $\chi^{2}$ test, the CUPI scoring system had the highest value (17.523), followed by the JIS system (15.819). In addition, the JIS system had the highest value in the 6-month c-index (0.659) as well as in the 1-year c-index (0.674). However, the CUPI classification system had the lowest value in the 1-year c-index (0.590). In conclusion, the JIS system may be an appropriate staging system for HCC patients undergoing sorafenib therapy.
\end{abstract}

Correspondence to: Dr Hiroki Nishikawa, Department of Gastroenterology and Hepatology, Osaka Red Cross Hospital, 5-30 Fudegasaki-cho, Tennoji-ku, Osaka, Osaka 543-0027, Japan

E-mail: h-nishikawa@osaka-med.jrc.or.jp

${ }^{*}$ Contributed equally

Key words: hepatocellular carcinoma, sorafenib, prognostic systems, predictability

\section{Introduction}

The design of a tumor staging system depends on the identification of individual predictors of survival in cancer patients (1-15). The staging of hepatocellular carcinoma (HCC) differs significantly from that of other malignancies, as the underlying liver disease, apart from the biology of the tumor itself, may significantly affect patient prognosis (1-15). Based on the identification of relevant predictors for tumor burden and liver functional reserve, several staging systems for HCC including both aspects have been proposed in different parts of the world (1-15). Of these prognostic systems for HCC, the Japan Integrated Staging (JIS) system, the Barcelona Clinic Liver Cancer (BCLC) classification system, the tumor-node-metastasis (TNM) classification system, the Cancer of the Liver Italian Program (CLIP) scoring system and the Chinese University Prognostic Index (CUPI) scoring system are currently used in daily clinical practice, with an ongoing debate between Western and Eastern countries regarding their prognostic ability in $\operatorname{HCC}(2,6,10-12,14)$.

Sorafenib (Nexavar; Bayer Healthcare Pharmaceuticals, Montville, NJ, USA), a multikinase inhibitor that blocks tumor growth and cell proliferation, was the first systemic chemotherapeutic agent found to significantly improve the survival of patients with advanced HCC in the Sorafenib HCC Assessment Randomised Protocol (SHARP) trial and in the Asian Pacific trial, and it is currently approved for use as first-line systemic chemotherapy in these patients $(16,17)$. In order to optimize the beneficial effects of sorafenib, combination or sequential therapies comprising sorafenib and other HCC therapies, such as transcatheter arterial chemoembolization (TACE), were recently investigated (18). However, to the best of our knowledge, predictive factors of responders to sorafenib among HCC patients have not been well established, and none of the prognostic staging systems for HCC patients who underwent sorafenib therapy is yet universally adopted or preferred $(19,20)$. Thus, there is an urgent need for determining the prognostic ability of staging systems in patients with advanced $\mathrm{HCC}$ receiving sorafenib therapy.

The aim of the present study was to compare prognostic ability among the five aforementioned well-known prognostic systems (JIS, BCLC, TNM, CLIP and CUPI systems) for HCC patients who received sorafenib therapy. 


\section{Patients and methods}

Patients. A total of 143 HCC patients were treated with sorafenib monotherapy at the Osaka Red Cross Hospital (Osaka, Japan) between June, 2009 and 2014. Subjects participating in clinical trials of novel molecular targeted agents or sequential or combination therapies with TACE and sorafenib were excluded from the present analysis. Sorafenib therapy was indicated in patients with unresectable HCC determined by dynamic computed tomography (CT): i) Eastern Cooperative Oncology Group performance status (ECOG PS) of 0-2; ii) presence of extrahepatic metastases; iii) $\mathrm{HCC}$ refractory to previous therapies, such as TACE; iv) unsuitability for TACE due to anatomical reasons; or v) vascular invasion, such as tumor thrombus in the portal vein $(19,21)$.

The disease was staged for all analysed patients by means of five staging systems, including the JIS, BCLC, TNM, CLIP and CUPI systems $(2,6,10,11,14)$. We investigated the prognostic ability of each prognostic system. Furthermore, we investigated prognostic factors associated with overall survival (OS) using univariate and multivariate analyses. The following data were used for the analyses: gender, age, tumor burden, presence of portal vein invasion, presence of extrahepatic metastases, Child-Pugh classification, ECOG PS, cause of liver disease, aspartate aminotransferase, alanine aminotransferase, alkaline phosphatase (ALP), platelet count, tumor markers and initial dose of sorafenib [recommended (800 mg/day) or reduced dose].

Prior to sorafenib therapy for HCC, written informed consent for HCC therapy was obtained from all the subjects. The Ethics Committee of our department approved the study protocol. The present study comprised a retrospective analysis of patients' medical records in our database and all the treatments were performed in an open-label manner.

Diagnosis of HCC and sorafenib therapy. $\mathrm{HCC}$ was diagnosed based on the results of the abdominal ultrasound and dynamic CT scan (hyperattenuation during the arterial phase in the entire or part of the tumor, and hypoattenuation in the portal venous phase) and/or magnetic resonance imaging (MRI), mainly as recommended by the American Association for the Study of Liver Diseases (22). Arterial and portal phase dynamic CT images were obtained $\sim 30$ and $120 \mathrm{sec}$ after injection of contrast material. In our hospital, abdominal angiography combined with CT (angio-CT) was routinely performed prior to therapy for HCC after obtaining informed consent from the patients. This was performed based on the fact that this technique was useful for detecting small satellite nodules, as reported by Yamasaki et al (23). Subsequently, HCC was confirmed using CT during hepatic arteriography and during arterial portography. Patients who presented with atypical liver tumors underwent ultrasound-guided tumor biopsy. Vascular invasion was determined using dynamic $\mathrm{CT}$ and/or angio-CT. During initial evaluation for $\mathrm{HCC}$, a chest $\mathrm{X}$-ray was performed and, if abnormal, it was followed by a chest CT scan. Bone scintigraphy, brain CT or MRI was performed if there were any symptoms or clinical indications.

The response to sorafenib was assessed every 4-8 weeks after the initiation of sorafenib therapy, using the modified Response Evaluation Criteria in Solid Tumors (mRECIST) and/or tumor markers (24). Sorafenib therapy was continued until disease progression, unacceptable drug-related toxicity, or the patient's wish to discontinue treatment. After discontinuation of sorafenib therapy for any reason, any additional therapies, such as TACE or systemic chemotherapy, were allowed based on the status of each patient $(19,21)$.

As regards the initial dose of sorafenib, for patients without risk factors, we introduced the recommended initial dose of $400 \mathrm{mg}$ twice a day $(800 \mathrm{mg} /$ day $)$ of sorafenib $(16,17,19,21,25,26)$. Considering previous studies on dose reduction of sorafenib, the initial dose was reduced based on clinical factors such as age, body weight, ECOG PS and liver functional reserve $(19,21,27)$. During sorafenib therapy, each attending physician decided to reduce the daily dose of sorafenib according to the grades of adverse events or ECOG PS. Sorafenib-related toxicities, including hand-foot skin reaction (HFSR), rash, diarrhea, fever, hypertension, fatigue, liver injury, gastrointestinal bleeding and lung injury were evaluated using the Common Terminology Criteria for Adverse Events (CTCAE) version 3.0 (http://ctep.cancer.gov).

Statistical analysis. In this study, OS was the only endpoint. Data were analysed using univariate and multivariate methods. To analyse the significance of prognostic predictors, continuous variables were divided by the median values for all cases $(n=143)$ and treated as dichotomous covariates. The cumulative OS rate was calculated by the Kaplan-Meier method and tested by the log-rank test. A Cox proportional hazards model via a stepwise forward method was used for multivariate analyses of factors with a P-value of $<0.05$ in the univariate analysis. These statistical methods were used to estimate the interval from each date of initiation of sorafenib therapy for HCC until the date of death or last follow-up.

The performance of a prognostic system has been demonstrated to be related to homogeneity (small differences in survival among subjects in the same stage within each system), monotonicity of gradients (the survival of subjects in more advanced stages is shorter compared with the survival of subjects in earlier stages within the same system) and discriminatory ability (greater differences in survival among subjects in different stages within each system) (28). The prognostic performance of each scoring system was statistically evaluated by homogeneity within classification groups, monotonicity of the gradients and discriminatory ability in the association between stage and survival rate. Homogeneity was determined by the likelihood ratio (LR) $\chi^{2}$ test based on a Cox proportional hazards regression model (28). Monotonicity of gradients was evaluated by the linear trend $\chi^{2}$ test using a Cox regression model (28). To evaluate the discriminatory ability for predicting survival, we assessed the accuracy of prediction of death at 6 months and 1 year for each scoring system. This score was assessed by calculating the area under the receiver operating characteristic curve for each score, which is equivalent to the concordance index (c-index) (29). To perform this test, subjects censored prior to 6 months or 1 year were excluded from the analysis. The c-index ranges between 0.0 and 1.0; a c-index of 0.5 indicates that the model is no better than chance at making a prediction of membership in a group, whereas a value of 1.0 indicates that the model perfectly identifies those within a group and those not. Models are typically considered 
Table I. Baseline characteristics ( $n=143)$.

\begin{tabular}{|c|c|}
\hline Characteristics & $\begin{array}{l}\text { No. or median } \\
\text { value (range) }\end{array}$ \\
\hline Age (years) & $71(45-89)$ \\
\hline Gender, male/female & $114 / 29$ \\
\hline \multicolumn{2}{|l|}{ Causes of liver disease } \\
\hline Hepatitis $\mathrm{B} / \mathrm{C} /$ non-B, non-C/B+C & $22 / 85 / 32 / 4$ \\
\hline Child-Pugh class, A/B & $102 / 41$ \\
\hline ECOG PS $0 / 1 / 2$ & $119 / 19 / 5$ \\
\hline Tumor burden, $<50 / \geq 50 \%$ & $129 / 14$ \\
\hline Portal vein invasion, present/absent & $31 / 112$ \\
\hline Extrahepatic metastases, present/absent & $63 / 80$ \\
\hline AST (IU/l) & $52(17-791)$ \\
\hline ALT (IU/1) & $34(7-380)$ \\
\hline ALP (IU/1) & $405(162-4535)$ \\
\hline Total bilirubin (mg/dl) & $0.8(0.3-2.5)$ \\
\hline Serum albumin $(\mathrm{g} / \mathrm{dl})$ & $3.4(1.7-4.8)$ \\
\hline Prothrombin time $(\%)$ & $79(48-116)$ \\
\hline Platelet count $\left(\mathrm{x} 10^{4} / \mathrm{mm}^{3}\right)$ & $11.5(3.4-29.5)$ \\
\hline $\mathrm{AFP}(\mathrm{ng} / \mathrm{ml})$ & $139.1(1.8-688,400)$ \\
\hline $\mathrm{DCP}(\mathrm{mAU} / \mathrm{ml})^{\mathrm{a}}$ & $1,341(10-421,210)$ \\
\hline $\begin{array}{l}\text { Initial dose of sorafenib (mg/day), } \\
800 / 400 / 200\end{array}$ & $35 / 106 / 2$ \\
\hline
\end{tabular}

${ }^{a}$ Missing data, $n=3$. ECOG PS, Eastern Cooperative Oncology Group performance status; AST, aspartate aminotransferase; ALT, alanine aminotransferase; ALP, alkaline phosphatase; AFP, $\alpha$-fetoprotein; DCP, des- $\gamma$-carboxy prothrombin.

reasonable when the c-index is $>0.70$ (30). In conclusion, the higher values of the LR $\chi^{2}$ test, linear trend $\chi^{2}$ test and c-index indicate that the prognostic system is more informative.

Data were analysed using SPSS software version 21 (SPSS Inc., Chicago, IL, USA) for Microsoft Windows and are expressed as median value (range). A P-value of $<0.05$ was considered to indicate statistically significant differences.

\section{Results}

Patient demographic characteristics. The baseline demographic characteristics of the analysed patients $(n=143)$ are listed in Table I. The patients included 114 men and 29 women, with a median age of 71 years (range, $45-89$ years). A total of 102 patients were classed as Child-Pugh A and 41 as Child-Pugh B. In terms of ECOG PS, 119, 19 and 5 subjects had a PS score of 0,1 and 2 , respectively. A total of 31 patients $(21.7 \%)$ had portal vein invasion and $63(44.1 \%)$ had extrahepatic metastases. The proportion of viral hepatitis (hepatitis B, C or B+C)-related HCC was $77.6 \%$ (111/143). In the present analysis, des- $\gamma$-carboxy prothrombin (DCP) data were missing from 3 subjects.

As regards previous therapies for HCC, the majority of our cohort $(90.9 \%, 130 / 143)$ underwent $\geq 1$ sessions of TACE for HCC prior to sorafenib therapy. Percutaneous ablative therapies, such as radiofrequency ablation or percutaneous ethanol

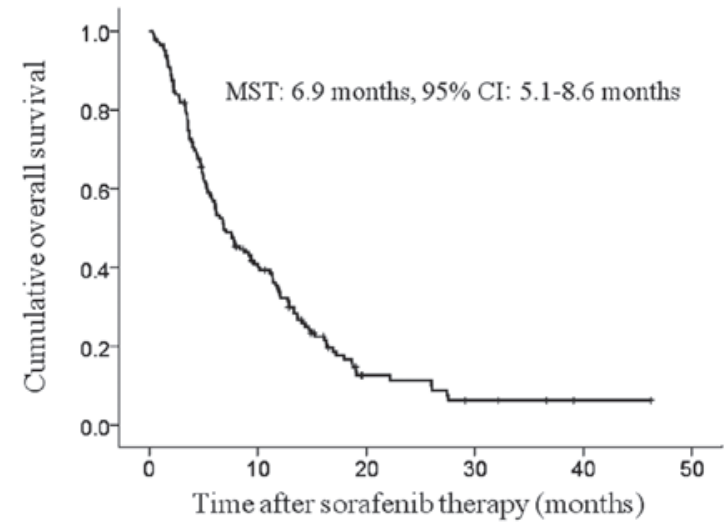

Figure 1. Cumulative overall survival for all cases $(n=143)$. The median survival time (MST) was 6.9 months (95\% CI: 5.1-8.6 months).

injection, were performed in $73(51.0 \%)$ and surgical resection in $33(23.1 \%)$ patients.

Overall survival and causes of death for all cases. The median follow-up period was 6.8 months (range, 0.3-46.2 months) and the median survival time (MST) was 6.9 months (95\% CI: 5.1-8.6 months) (Fig. 1). During the follow-up period, there were $121(84.6 \%)$ deaths. The causes of death were HCC progression in 97 patients, liver failure in 4, sorafenib-related serious adverse events (SAE) in 1 and miscellaneous causes in 19 patients.

Best treatment response, dose adjustment or discontinuation, sorafenib-related adverse events and therapy after sorafenib discontinuation. During sorafenib therapy, the best treatment responses according to the mRECIST were as follows: complete response in 2, partial response in 10, stable disease in 44, progressive disease in 51 and not evaluated in 36 patients (24).

In patients treated with the standard initial dose of sorafenib ( $800 \mathrm{mg} / \mathrm{day}, \mathrm{n}=35)$, dose reduction was performed in 15 patients during sorafenib therapy. In patients treated with a reduced initial dose of sorafenib (400 or $200 \mathrm{mg} / \mathrm{day}, \mathrm{n}=108$ ), dose escalation of sorafenib was performed in 25 and dose reduction in 22 patients during sorafenib therapy. Overall, the treatment discontinuation rate was $93.7 \%$ (134/143).

In terms of sorafenib-related grade $\geq 3$ SAEs according to the CTCAE 3.0, rash was observed in 4 patients, HFSR in 8 , diarrhea in 7, gastrointestinal bleeding in 4, liver injury in 33, general fatigue in 7, fever in 6 and lung injury in 3 patients.

As regards HCC therapy after sorafenib discontinuation, $\geq 1$ sessions of TACE were performed in 29 patients, while chemotherapeutic agents other than sorafenib were administered in 21 patients based on liver function or PS.

Univariate and multivariate analyses of factors contributing to $O S$. On the univariate analysis of factors affecting OS, gender $(\mathrm{P}=0.002)$, tumor burden $(\mathrm{P}=0.007)$, extrahepatic metastases $(\mathrm{P}=0.001)$, Child-Pugh classification $(\mathrm{P}=0.007)$ and $\mathrm{DCP}>1,341 \mathrm{mAU} / \mathrm{ml}(\mathrm{P}=0.018)$ were found to be significant factors associated with OS (Table II). The multivariate analysis involving five factors with $\mathrm{P}<0.05$ in the univariate analysis demonstrated that gender $(\mathrm{P}=0.003)$, tumor burden 
Table II. Univariate and multivariate analyses of factors contributing to overall survival ( $\mathrm{n}=143)$.

\begin{tabular}{|c|c|c|c|c|}
\hline \multirow[b]{2}{*}{ Variables } & \multirow[b]{2}{*}{$\mathrm{n}$} & \multirow{2}{*}{$\begin{array}{c}\text { Univariate } \\
\text { analysis }\end{array}$} & \multicolumn{2}{|c|}{ Multivariate analysis } \\
\hline & & & Hazard ratio $(95 \% \mathrm{CI})$ & P-value ${ }^{a}$ \\
\hline Gender, male vs. female & $114 / 29$ & 0.002 & $2.231(1.320-3.770)$ & 0.003 \\
\hline Age, $>71$ vs. $<71$ years & $75 / 68$ & 0.742 & & \\
\hline Tumor burden, $<50$ vs. $>50 \%$ & $129 / 14$ & 0.007 & $0.381(0.207-0.702)$ & 0.002 \\
\hline Portal vein invasion, yes vs. no & $31 / 112$ & 0.985 & & \\
\hline Extrahepatic metastases, yes vs. no & $63 / 80$ & 0.001 & $2.273(1.546-3.333)$ & $<0.001$ \\
\hline Child-Pugh class, A vs. B & $102 / 41$ & 0.007 & $0.508(0.335-0.771)$ & 0.001 \\
\hline ECOG PS, 0 vs. $>1$ & $119 / 24$ & 0.278 & & \\
\hline Cause of liver disease, virus-related vs. NBNC & $111 / 32$ & 0.844 & & \\
\hline $\mathrm{AST},>52$ vs. $<52 \mathrm{IU} / 1$ & $72 / 71$ & 0.234 & & \\
\hline ALT, $>34$ vs. $<34$ IU/1 & $75 / 68$ & 0.476 & & \\
\hline ALP, $>405$ vs. $<405 \mathrm{IU} / 1$ & $72 / 71$ & 0.221 & & \\
\hline Platelet count, $>11.5$ vs. $<11.5 \times 10^{4} / \mathrm{mm}^{3}$ & $72 / 71$ & 0.492 & & \\
\hline AFP, $>139.1$ vs. $<139.1 \mathrm{ng} / \mathrm{ml}$ & $72 / 71$ & 0.959 & & \\
\hline DCP, $>1,341$ vs. $<1,341 \mathrm{mAU} / \mathrm{ml}^{\mathrm{b}}$ & $70 / 70$ & 0.018 & & \\
\hline Initial dose of sorafenib, $800 \mathrm{mg} /$ day vs. reduced dose & $35 / 108$ & 0.665 & & \\
\hline
\end{tabular}

${ }^{a}$ Cox proportional hazards model. ${ }^{b}$ Missing data, $n=3$. CI, confidence interval; ECOG PS, Eastern Cooperative Oncology Group performance status; NBNC, non-B, non-C; AST, aspartate aminotransferase; ALT, alanine aminotransferase; ALP, alkaline phosphatase; AFP, $\alpha$-fetoprotein; DCP, des- $\gamma$-carboxy prothrombin.

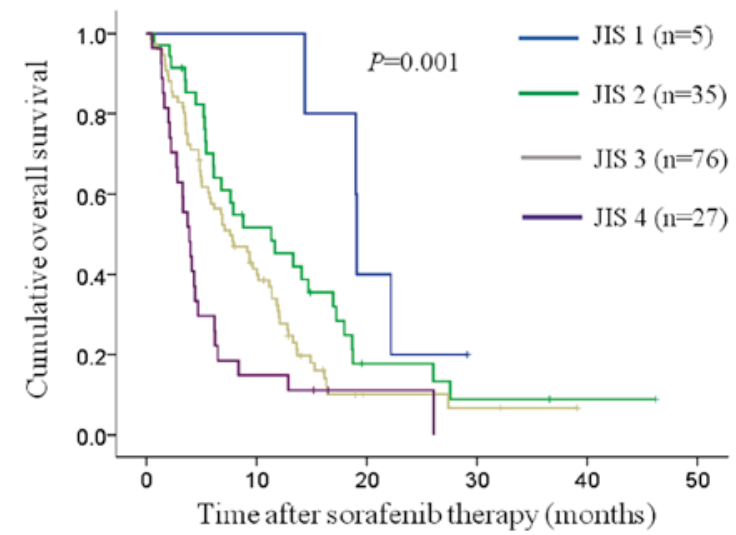

Figure 2. Kaplan-Meier survival curves for hepatocellular carcinoma patients treated with sorafenib according to the Japan Integrated Staging (JIS) system (overall significance, $\mathrm{P}=0.001$ ).

$(\mathrm{P}=0.002)$, extrahepatic metastases $(\mathrm{P}<0.001)$ and Child-Pugh classification $(\mathrm{P}=0.001)$ were significant independent predictors associated with OS. Of note, gender was a significant predictor that was not included in different staging systems. The hazard ratios (HRs), 95\% confidence intervals (CIs) and $\mathrm{P}$-values for these factors are listed in Table II.

Comparison offive prognostic systems for all cases ( $n=143)$ using the LR $\chi^{2}$ test, linear trend $\chi^{2}$ test and c-index. Kaplan-Meier curves of OS were constructed for the JIS, BCLC, TNM, CLIP and CUPI systems (Figs. 2-6). The number and median OS of patients with each score are presented in Table III. The P-values between adjacent groups in each system are also shown in Table III. The overall significance in all prognostic systems

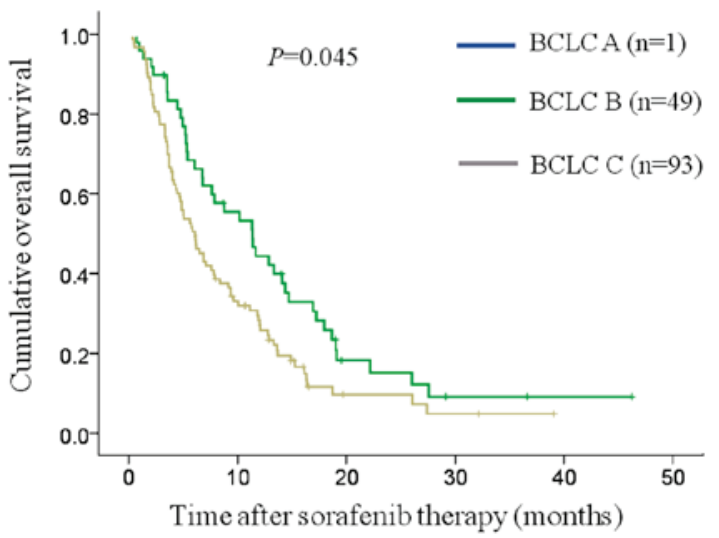

Figure 3. Kaplan-Meier survival curves for hepatocellular carcinoma patients treated with sorafenib according to the Barcelona Clinic Liver Cancer (BCLC) classification system (overall significance, $\mathrm{P}=0.045$ ).

was $\mathrm{P}<0.05$. The differences between adjacent groups reached statistical significance: In the JIS system, between JIS 3 and 4 $(\mathrm{P}=0.013)$; in the BCLC classification system, between BCLC B and $\mathrm{C}(\mathrm{P}=0.017)$; in the TNM classification system, between stages III and IV $(\mathrm{P}=0.007)$; and in the CUPI scoring system, between the low-and intermediate-risk groups $(\mathrm{P}=0.005)$ and between the high- and intermediate-risk groups $(\mathrm{P}=0.001)$.

Using the LR $\chi^{2}$ test, the CUPI classification system had the highest value $(35.804, \mathrm{P}<0.001)$ among the five prognostic systems, followed by the JIS system $(17.469, \mathrm{P}=0.001)$, indicating small differences in survival among subjects in the same stages of these two groups (Table IV). Using the linear trend $\chi^{2}$ test, the CUPI scoring system had the highest value (17.523), followed by the JIS system (15.819), indicating that these two 
Table III. Patient survival according to different staging systems.

\begin{tabular}{|c|c|c|c|c|}
\hline Staging system & MST (months) & $95 \% \mathrm{CI}$ & P-value (overall) & $\begin{array}{l}\mathrm{P} \text {-value in each } \\
\text { adjacent group }\end{array}$ \\
\hline JIS system & & & 0.001 & \\
\hline $1(\mathrm{n}=5)$ & 19.1 & $19.0-19.2$ & & 1 vs. $2,0.132$ \\
\hline $2(\mathrm{n}=35)$ & 11.3 & $5.2-17.5$ & & 2 vs. $3,0.088$ \\
\hline $3(n=76)$ & 7.6 & $5.2-10.0$ & & 3 vs. $4,0.013$ \\
\hline $4(n=27)$ & 3.9 & $2.8-5.0$ & & \\
\hline BCLC classification system & & & 0.045 & \\
\hline A (early stage, $n=1)$ & NT & NT & & \\
\hline B (intermediate stage, $n=49$ ) & 11.4 & $7.6-15.1$ & & B vs. C, 0.017 \\
\hline $\mathrm{C}$ (advanced stage, $\mathrm{n}=93$ ) & 6.1 & $4.5-7.7$ & & \\
\hline TNM classification system & & & 0.007 & \\
\hline Stage II $(n=7)$ & 19.0 & $7.1-31.0$ & & II vs. III, 0.336 \\
\hline Stage III $(n=45)$ & 11.4 & $6.5-16.3$ & & III vs. IV, 0.007 \\
\hline Stage IV (n=91) & 5.7 & $4.5-7.0$ & & \\
\hline CLIP scoring system & & & 0.038 & \\
\hline $1(n=54)$ & 11.8 & $7.7-15.9$ & & 1 vs. $2,0.315$ \\
\hline $2(n=48)$ & 6.1 & $3.3-8.9$ & & 2 vs. $3,0.117$ \\
\hline $3(n=31)$ & 4.1 & $1.2-7.1$ & & 3 vs. $4,0.895$ \\
\hline $4(n=10)$ & 4.3 & $2.8-5.9$ & & \\
\hline CUPI scoring system & & & $<0.001$ & \\
\hline Low-risk group $(\mathrm{L})(\mathrm{n}=106)$ & 9.4 & $6.3-12.4$ & & L vs. I, 0.005 \\
\hline Intermediate-risk group (I) $(n=33)$ & 4.4 & $3.6-5.2$ & & I vs. H, 0.001 \\
\hline High-risk group $(\mathrm{H})(\mathrm{n}=4)$ & 1.5 & $0.7-2.3$ & & \\
\hline
\end{tabular}

JIS, Japan Integrated Staging; BCLC, Barcelona Clinic Liver Cancer; TNM, tumor-node-metastasis; CLIP, Cancer of the Liver Italian Program; CUPI, Chinese University Prognostic Index; MST, median survival time; CI, confidence interval; NT, not tested.

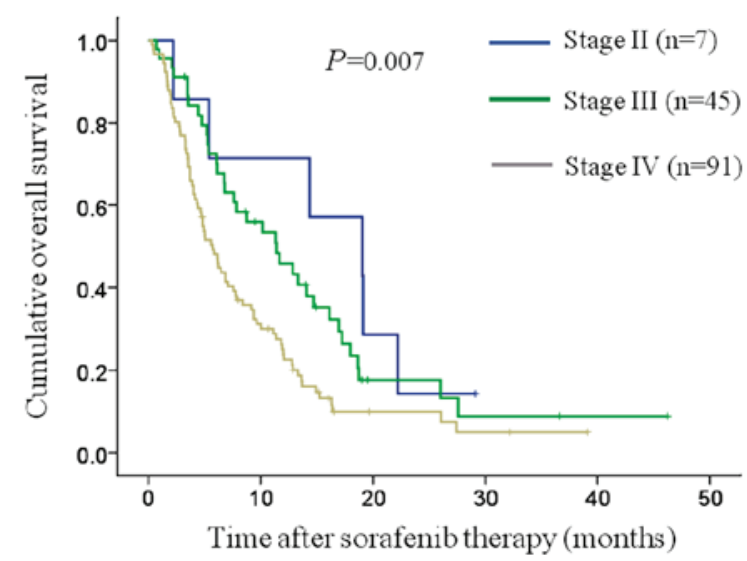

Figure 4. Kaplan-Meier survival curves for hepatocellular carcinoma patients treated with sorafenib according to the tumor-node-metastasis classification system (overall significance, $\mathrm{P}=0.007$ ).

prognostic systems gave an accurate prediction of patient survival (monotonicity of the prognostic system) (Table IV). Using the c-index, the JIS system had the highest value at 6 months (0.659) and 1 year (0.674), suggesting that the JIS system had the highest discriminative ability among the five prognostic systems (Table V).
Table IV. Values of LR $\chi^{2}$ test and linear trend $\chi^{2}$ test in each prognostic system.

\begin{tabular}{lrcc}
\hline Systems & LR $\chi^{2}$ test & P-value & Linear trend $\chi^{2}$ test \\
\hline JIS & 17.469 & 0.001 & 15.819 \\
BCLC & 6.138 & 0.013 & 6.162 \\
TNM & 9.470 & 0.002 & 9.505 \\
CLIP & 7.891 & 0.005 & 7.922 \\
CUPI & 35.804 & $<0.001$ & 17.523 \\
\hline
\end{tabular}

LR, likelihood ratio; JIS, Japan Integrated Staging; BCLC, Barcelona Clinic Liver Cancer; TNM, tumor-node-metastasis; CLIP, Cancer of the Liver Italian Program; CUPI, Chinese University Prognostic Index.

\section{Discussion}

In the present analysis, in terms of homogeneity and monotonicity of gradients, the CUPI scoring system had the highest values among the five prognostic systems, followed by the JIS system. In terms of discriminative ability, the JIS system had the highest c-index at the time points of 6 months and 1 year, while the CUPI scoring system had the lowest c-index at the 1-year time point. The ideal cancer staging system must 
Table V. Comparison of discriminative ability using 6 months and 1-year concordance index (c-index) among five prognostic systems .

\begin{tabular}{|c|c|c|c|c|c|c|}
\hline \multirow[b]{2}{*}{ Systems } & \multicolumn{3}{|c|}{6 months } & \multicolumn{3}{|c|}{1 year } \\
\hline & c-index & $95 \% \mathrm{CI}$ & P-value & c-index & $95 \% \mathrm{CI}$ & P-value \\
\hline JIS & 0.659 & $0.568-0.749$ & 0.001 & 0.674 & $0.573-0.775$ & 0.001 \\
\hline BCLC & 0.580 & $0.485-0.674$ & 0.107 & 0.606 & $0.500-0.712$ & 0.051 \\
\hline TNM & 0.613 & $0.519-0.706$ & 0.022 & 0.658 & $0.553-0.762$ & 0.004 \\
\hline CLIP & 0.627 & $0.534-0.720$ & 0.010 & 0.628 & $0.528-0.728$ & 0.018 \\
\hline CUPI & 0.634 & $0.539-0.729$ & 0.007 & 0.590 & $0.490-0.689$ & 0.099 \\
\hline
\end{tabular}

JIS, Japan Integrated Staging; BCLC, Barcelona Clinic Liver Cancer; TNM, tumor-node-metastasis; CLIP, Cancer of the Liver Italian Program; CUPI, Chinese University Prognostic Index; CI, confidence interval.

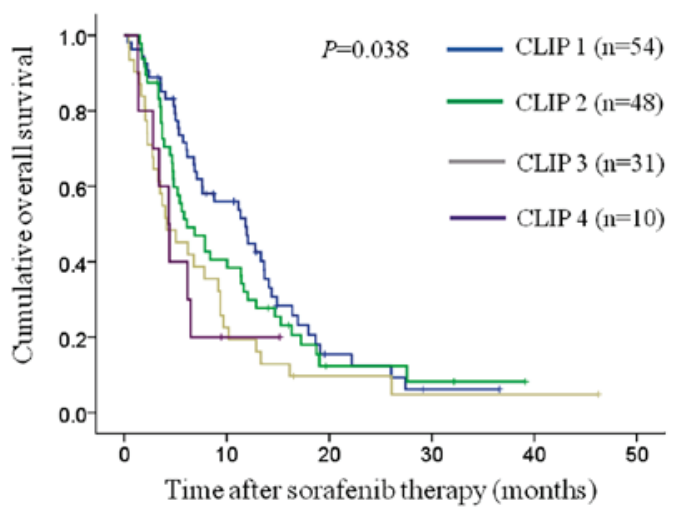

Figure 5. Kaplan-Meier survival curves for hepatocellular carcinoma patients treated with sorafenib according to the Cancer of the Liver Italian Program (CLIP) scoring system (overall significance, $\mathrm{P}=0.038$ ).

provide maximal discrimination of clinical outcomes among different stages of the disease, while maintaining the variability of outcomes within each stage to a minimum (31). In view of our present results, the JIS system may be the most appropriate among the five prognostic systems for HCC patients undergoing sorafenib therapy. The JIS system was introduced in Japan, whereas the CUPI scoring system was introduced in China $(10,14)$. The major difference in the HCC characteristics between these two Asian countries is the main etiology of liver disease: In Japan it is chronic hepatitis $\mathrm{C}$ virus infection, whereas in China it is chronic hepatitis B virus infection $(10,14)$. However, these differences may not affect survival of patients with advanced HCC who received sorafenib therapy.

In our results, the values of the LR $\chi^{2}$ test, linear trend $\chi^{2}$ test and 6-month c-index in the BCLC classification were the lowest among the five systems. Although the BCLC classification system is still widely used and is the most comprehensive staging system available, previous studies have demonstrated that the performance of the BCLC classification system may be better in Caucasian HCC patients and earlier-stage disease only $(6-9,13,32)$. Our data were consistent with these findings.

The TNM classification system was inferior to JIS and CUPI in terms of homogeneity, monotonicity and discriminative ability, although at 1 year the value of the c-index for TNM was the second highest in the present study. JIS was based on

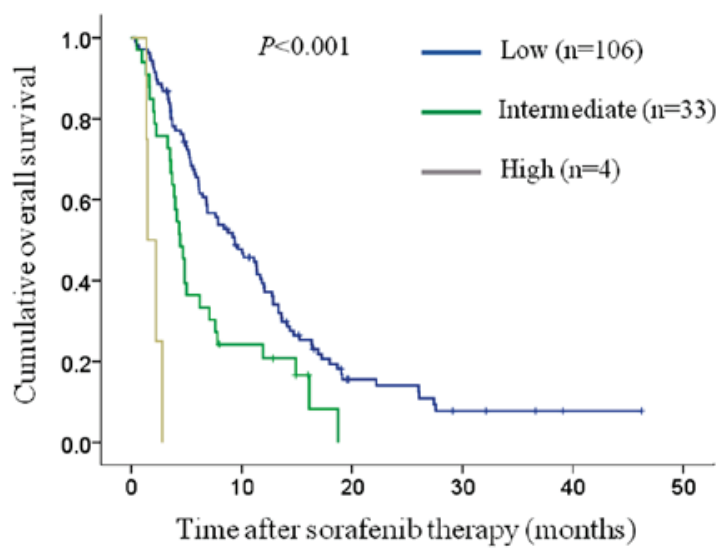

Figure 6. Kaplan-Meier survival curves for hepatocellular carcinoma patients treated with sorafenib according to the Chinese University Prognostic Index scoring system (overall significance, $\mathrm{P}<0.001$ ).

TNM, followed by the addition of liver function, whereas CUPI was based on TNM, followed by the addition of liver function and symptom evaluation in the risk stratification $(10,11,14)$. In advanced HCC patients, factors other than tumor-related factors may be essential for risk stratification $(2,10,11,14)$. Similarly, the CLIP scoring system was inferior to JIS and CUPI in our results. The CLIP scoring system uses portal vein invasion as a marker of tumor extension (2). However, in our analysis, patients with portal vein invasion had an almost identical prognosis compared with those without portal vein invasion in our univariate analysis $(\mathrm{P}=0.985)$. These observation may be associated with our present results.

In our data, the MST was 6.9 months, which is shorter compared with that in the SHARP trial (10.7 months). This is probably due to the difference in the proportion of patients with Child-Pugh class B between our cohort $28.7 \%$ (41/143) and the SHARP study (5\%) (16). Of note, gender was a significant predictor associated with OS in the multivariate analysis, along with other well-known predictors $(\mathrm{P}=0.003)$. One possibility is genomic alterations, such as mutation or amplification in female HCC patients (33). However, we did not investigate these alterations in our cohorts; thus, further examination is required. However, the initial dose of sorafenib was not a significant predictor. The optimal dose of sorafenib 
for Japanese HCC patients with a relatively lower body weight compared with Western populations remains unclear (21) and further investigation is required.

We acknowledge several limitations to the present analysis. First, this was a single-center retrospective study including only Japanese HCC patients. Second, the initial dose of sorafenib varied among individual patients, leading to bias. Third, various therapies were applied after discontinuation of sorafenib, also potentially leading to bias regarding their OS. Therefore, our results must be interpreted with caution. Fourth, since any staging system is constructed from selected prognostic factors for a certain stage of HCC in a specific population, the predictive ability of the staging system may be considerably impaired if it is applied to another patient population and the clinical outcome is closely associated with patient characteristics. Thus, various staging systems for HCC patients undergoing sorafenib therapy should be compared in other independent populations $(7,34,35)$. Finally, there were several values missing from our study. However, our results demonstrated that the JIS system exhibited a high prognostic ability for HCC patients treated with sorafenib.

In conclusion, the JIS system may be a useful prognostic tool for patients undergoing sorafenib therapy.

\section{Acknowledgements}

We would like to thank Mrs. Haruko Takada for the data collection.

\section{References}

1. Huitzil-Melendez FD, Capanu M, O'Reilly EM, Duffy A, Gansukh B, Saltz LL and Abou-Alfa GK: Advanced hepatocellular carcinoma: Which staging systems best predict prognosis? J Clin Oncol 28: 2889-2895, 2010.

2. No authors listed: A new prognostic system for hepatocellular carcinoma: a retrospective study of 435 patients: the Cancer of the Liver Italian Program (CLIP) investigators. Hepatology 28: 751-755, 1998.

3. Poon D, Anderson BO, Chen LT, Tanaka K, Lau WY, Van Cutsem E, Singh H, Chow WC, Ooi LL, Chow P, et al; Asian Oncology Summit: Management of hepatocellular carcinoma in Asia: Consensus statement from the Asian Oncology Summit 2009. Lancet Oncol 10: 1111-1118, 2009.

4. Takanishi DM Jr, Severino R and Wong LL: The Cancer of the Liver Italian Program (CLIP) score: Validation of a new prognostic system for hepatocellular carcinoma. Hawaii Med J 66 : 209-212, 2007.

5. Levy I and Sherman M; Liver Cancer Study Group of the University of Toronto: Staging of hepatocellular carcinoma: Assessment of the CLIP, Okuda, and Child-Pugh staging systems in a cohort of 257 patients in Toronto. Gut 50: 881-885, 2002

6. Llovet JM, Brú C and Bruix J: Prognosis of hepatocellular carcinoma: The BCLC staging classification. Semin Liver Dis 19: 329-338, 1999.

7. Marrero JA, Fontana RJ, Barrat A, Askari F, Conjeevaram HS, Su GL and Lok AS: Prognosis of hepatocellular carcinoma: Comparison of 7 staging systems in an American cohort. Hepatology 41: 707-716, 2005 .

8. Cillo U, Vitale A, Grigoletto F, Farinati F, Brolese A, Zanus G, Neri D, Boccagni P, Srsen N, D'Amico F, et al: Prospective validation of the Barcelona Clinic Liver Cancer staging system. J Hepatol 44: 723-731, 2006.

9. Bruix J, Sherman M, Llovet JM, Beaugrand M, Lencioni R, Burroughs AK, Christensen E, Pagliaro L, Colombo M and Rodés J; EASL Panel of Experts on HCC; European Association for the Study of the Liver: Clinical management of hepatocellular carcinoma. Conclusions of the Barcelona-2000 EASL conference. J Hepatol 35: 421-430, 2001.
10. Kudo M, Chung $\mathrm{H}$ and Osaki Y: Prognostic staging system for hepatocellular carcinoma (CLIP score): Its value and limitations, and a proposal for a new staging system, the Japan Integrated Staging Score (JIS score). J Gastroenterol 38: 207-215, 2003.

11. No authors listed: Liver Cancer Study Group of Japan: The general rules for the clinical and pathological study of primary liver cancer. Jpn J Surg 19: 98-129, 1989.

12. Kudo M, Chung H, Haji S, Osaki Y, Oka H, Seki T, Kasugai H, Sasaki Y and Matsunaga T: Validation of a new prognostic staging system for hepatocellular carcinoma: The JIS score compared with the CLIP score. Hepatology 40: 1396-1405, 2004.

13. Gomaa AI, Hashim MS and Waked I: Comparing staging systems for predicting prognosis and survival in patients with hepatocellular carcinoma in Egypt. PLoS One 9: e90929, 2014.

14. Leung TW, Tang AM, Zee B, Lau WY, Lai PB, Leung KL, Lau JT, Yu SC and Johnson PJ: Construction of the Chinese University Prognostic Index for hepatocellular carcinoma and comparison with the TNM staging system, the Okuda staging system, and the Cancer of the Liver Italian Program staging system: A study based on 926 patients. Cancer 94: 1760-1769, 2002.

15. Henderson JM, Sherman M, Tavill A, Abecassis M, Chejfec G and Gramlich T: AHPBA/AJCC consensus conference on staging of hepatocellular carcinoma: Consensus statement. HPB Oxf 5: 243-250, 2003.

16. Llovet JM, Ricci S, Mazzaferro V, Hilgard P, Gane E, Blanc JF, de Oliveira AC, Santoro A, Raoul JL, Forner A, et al; SHARP Investigators Study Group: Sorafenib in advanced hepatocellular carcinoma. N Engl J Med 359: 378-390, 2008.

17. Cheng AL, Kang YK, Chen Z, Tsao CJ, Qin S, Kim JS, Luo R, Feng J, Ye S, Yang TS, et al: Efficacy and safety of sorafenib in patients in the Asia-Pacific region with advanced hepatocellular carcinoma: A phase III randomised, double-blind, placebo-controlled trial. Lancet Oncol 10: 25-34, 2009.

18. Abdel-Rahman O and Fouad M: Sorafenib-based combination as a first line treatment for advanced hepatocellular carcinoma: A systematic review of the literature. Crit Rev Oncol Hematol 91: $1-8,2014$

19. Takeda H, Nishikawa H, Osaki Y, Tsuchiya K, Joko K, Ogawa C, Taniguchi H, Orito E, Uchida Y and Izumi N; Japanese Red Cross Liver Study Group: Clinical features associated with radiological response to sorafenib in unresectable hepatocellular carcinoma: a large multicenter study in Japan. Liver Int 35: 1581-1589, 2015.

20. Llovet JM, Peña CE, Lathia CD, Shan M, Meinhardt G and Bruix J; SHARP Investigators Study Group: Plasma biomarkers as predictors of outcome in patients with advanced hepatocellular carcinoma. Clin Cancer Res 18: 2290-2300, 2012.

21. Nishikawa H, Osaki Y, Endo M, Takeda H, Tsuchiya K, Joko K, Ogawa C, Taniguchi H, Orito E, Uchida Y, et al; Japanese Red Cross Liver Study Group: Comparison of standard-dose and half-dose sorafenib therapy on clinical outcome in patients with unresectable hepatocellular carcinoma in field practice: A propensity score matching analysis. Int J Oncol 45: 2295-2302, 2014.

22. Bruix J and Sherman M; Practice Guidelines Committee, American Association for the Study of Liver Diseases: Management of hepatocellular carcinoma. Hepatology 42: 1208-1236, 2005.

23. Yamasaki T, Kurokawa F, Shirahashi H, Kusano N, Hironaka K and Okita K: Percutaneous radiofrequency ablation therapy with combined angiography and computed tomography assistance for patients with hepatocellular carcinoma. Cancer 91: 1342-1348, 2001.

24. Lencioni R and Llovet JM: Modified RECIST (mRECIST) assessment for hepatocellular carcinoma. Semin Liver Dis 30: 52-60, 2010.

25. Park JW, Amarapurkar D, Chao Y, Chen PJ, Geschwind JF, Goh KL, Han KH, Kudo M, Lee HC, Lee RC, et al: Consensus recommendations and review by an International Expert Panel on Interventions in Hepatocellular Carcinoma (EPOIHCC). Liver Int 33: 327-337, 2013.

26. Kudo M: Targeted therapy for liver cancer: Updated review in 2012. Curr Cancer Drug Targets 12: 1062-1072, 2012.

27. Shingina A, Hashim AM, Haque M, Suen M, Yoshida EM, Gill S, Donnellan F and Weiss AA: In a 'real-world', clinic-based community setting, sorafenib dose of $400 \mathrm{mg} /$ day is as effective as standard dose of $800 \mathrm{mg} /$ day in patients with advanced hepatocellular carcinoma, with better tolerance and similar survival. Can J Gastroenterol 27: 393-396, 2013. 
28. Ueno S, Tanabe G, Sako K, Hiwaki T, Hokotate H, Fukukura Y, Baba Y, Imamura Y and Aikou T: Discrimination value of the new western prognostic system (CLIP score) for hepatocellular carcinoma in 662 Japanese patients. Cancer of the Liver Italian Program. Hepatology 34: 529-534, 2001.

29. Hanley JA and McNeil BJ: The meaning and use of the area under a receiver operating characteristic (ROC) curve. Radiology 143: 29-36, 1982.

30. Pencina MJ and D'Agostino RB: Overall C as a measure of discrimination in survival analysis: Model specific population value and confidence interval estimation. Stat Med 23: 2109-2123, 2004.

31. Maetani S, Onodera H, Nishikawa T and Tobe T: Systematic computer-aided search of optimal staging system for colorectal cancer. J Clin Epidemiol 44: 285-291, 1991.

32. Grieco A, Pompili M, Caminiti G, Miele L, Covino M, Alfei B, Rapaccini GL and Gasbarrini G: Prognostic factors for survival in patients with early-intermediate hepatocellular carcinoma undergoing non-surgical therapy: Comparison of Okuda, CLIP, and BCLC staging systems in a single Italian centre. Gut 54 411-418, 2005
33. Zhu ZZ, Wang D, Cong WM, Jiang H, Yu Y, Wen BJ, Dong $\mathrm{H}$, Zhang X, Liu SF, Wang AZ, et al: Sex-related differences in DNA copy number alterations in hepatitis B virus-associated hepatocellular carcinoma. Asian Pac J Cancer Prev 13: 225-229, 2012.

34. Cillo U, Bassanello M, Vitale A, Grigoletto FA, Burra P, Fagiuoli S, D'Amico F, Ciarleglio FA, Boccagni P, Brolese A, et al: The critical issue of hepatocellular carcinoma prognostic classification: Which is the best tool available? J Hepatol 40: 124-131, 2004.

35. Collette S, Bonnetain F, Paoletti X, Doffoel M, Bouché O, Raoul JL, Rougier P, Masskouri F, Bedenne L and Barbare JC: Prognosis of advanced hepatocellular carcinoma: Comparison of three staging systems in two French clinical trials. Ann Oncol 19: $1117-1126,2008$ 